\title{
Experimental Investigation of Low-frequency Wayside Noise from High-speed Train
}

\author{
Hajime TAKAMI, Dr. Eng. \\ Senior Researcher, \\ Katsuhiro KIKUCHI, Dr. Eng. \\ Senior Researcher, \\ Aerodynamics Laboratory, Environmental Engineering Division
}

\begin{abstract}
The authors performed laboratory experiments and field measurements to investigate low-frequency noise generated in wayside environments from high-speed train running. The results indicated the following three types of low-frequency sound sources: pressure fields around the nose and tail parts of the train, low-frequency acoustic pressure waves aerodynamically caused by the train itself, and noise radiation from vibrating concrete railway viaducts. Measurements conducted in a higher-speed section revealed that the major sound source of low-frequency noise in the far field was aerodynamically generated unsteady flow, which is analogous to a line source.
\end{abstract}

Keywords: high-speed train, low-frequency noise, aerodynamic sound, concrete bridge noise

\section{Introduction}

It has been reported that, in addition to audible noise, low-frequency noise including infrasound is radiated from high-speed trains (known as Shinkansen in Japan) toward open wayside areas, and represents one of technological barrier to the future development of higherspeed vehicles [1]. The low-frequency noise referred to here is components with a frequency below $100 \mathrm{~Hz}$. In previous studies, we measured low-frequency wayside noise caused by trains in an open section running in the range of $240-340 \mathrm{~km} / \mathrm{h}$. Measurement was carried out based on a manual issued by Japan's Ministry of the Environment (MOE), representing the first official document in Japan to specify the method of measuring lowfrequency noise [2]. Though the low-frequency wayside noise measured was relatively weak, it is expected to become stronger as train speeds increase.

These previous measurements indicated that two types of noise source are significant in low-frequency wayside noise, as shown in Fig. 1. The first consists of pressure variations caused by a train moving with quasisteady pressure fields around its nose and tail parts, referred to as passing-train pressure fields (hereafter $P_{f}$ ). This phenomenon has been well investigated analytically using potential theory [3]. The other is referred to as lowfrequency acoustic pressure waves (hereafter $P_{w}$ ), which have frequencies between 20 and $100 \mathrm{~Hz}$. Though past investigation suggested that this phenomenon was related to unsteady disturbed airflow around the train and the vibrating concrete structures of railway viaducts, the exact cause was not understood [4].

In this study, to provide a deeper understanding of $P_{w}$ phenomenon, field measurements were performed at several types of sites. Furthermore, in order to identify the aerodynamic characteristics of low-frequency wayside noise, laboratory experiments using an axisymmetric model were conducted in a higher speed range of $300-$ $500 \mathrm{~km} / \mathrm{h}$. Comparison between the field and laboratory measurements was made, enabling significant conclusions to be drawn.

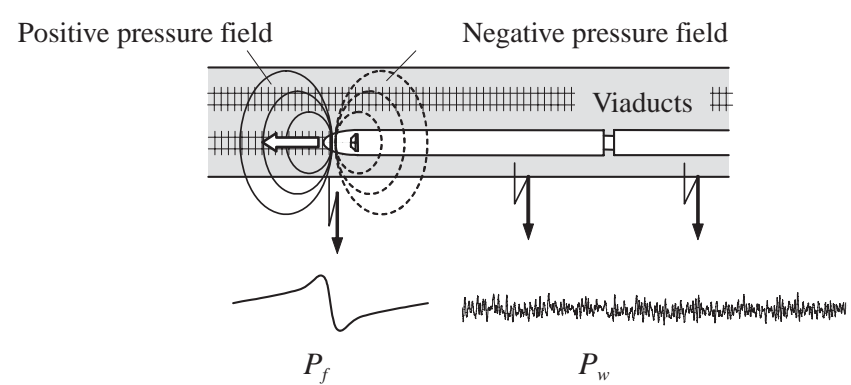

Fig. 1 Schematic view of low-frequency noise radiated from a train. $P_{f}$ represents pressure variations caused by moving pressure fields around the nose and tail parts of the vehicle. $P_{w}$ represents lowfrequency acoustic pressure waves caused by unsteady disturbed flow around the train and the vibrating structures of viaducts.

\section{Simple scale-model aerodynamic experiment}

The pressure variation of $P_{f}$ is modeled by a moving monopole given by [3]

$$
P_{f}(\mathbf{x}, t)=-\frac{\rho_{0} U^{2} A_{0}}{2 \pi} \frac{\cos (\mathbf{x}, \mathbf{y})}{r^{2}},
$$

where $\rho_{0}$ is the mean air density, $U$ is the train speed, $A_{0}$ is the cross-sectional area of the train, $\cos (\mathbf{x}, \mathbf{y})$ is the cosine of the angle of the monopole source point $\mathbf{y}$ at time $t$ relative to observation point $\mathbf{x}$, and $r(=|\mathbf{x}-\mathbf{y}|)$ is the distance from the observation point to the monopole source point. Equation (1) shows that the magnitude of $P_{f}$ is inversely proportional to the square of distance $r$, and is proportional to the square of train speed $U$.

The acoustic source mechanisms of $P_{w}$ are considered to relate to noise radiation from concrete railway viaducts (hereafter $P_{w, s}$ ) and the unsteady disturbed airflow around the train (hereafter $P_{w, a}$ ). The magnitude of $P_{w, s}$ depends on the type of viaduct structure, the weight of the train and the roughness of the rail/wheel treads. Under regular conditions of these variables, the magnitude of $P_{w, s}$ is empirically given by [5] 


$$
P_{w, S} \propto \frac{U^{1.5}}{\sqrt{r}} .
$$

No theoretical/empirical formula exists for the magnitude of $P_{w, a}$ because of the lack of an understanding about the exact cause. Assuming that the main source of $P_{w, a}$ is the unsteady disturbed airflow at the whole surface of the car body rather than at specific parts, we estimate the characteristic of $P_{w, a}$ by the following laboratory experiments using an axisymmetric model; these experiments can be performed in a high speed range without noise from railway viaducts.

\subsection{Wind-tunnel experiment}

The experiments reported here were carried out using a simple scale-model train at about 1/110 of full scale train. The model had an axisymmetric body with ellipsoidal nose and tail profiles and the dimensions given in Table 1. The model did not cause Aeolian tones or cavity tones due to its smooth surface without projections or gaps.

A preliminary experiment was performed to evaluate the statistical values of the boundary layer around the model surface using the closed-circuit wind tunnel facility at the Railway Technical Research Institute (RTRI). The experimental apparatus is shown in Fig. 2. The test section has a cross-section width of $720 \mathrm{~mm}$ and a height of $600 \mathrm{~mm}$, and measures $1.5 \mathrm{~m}$ in length. The experiment was conducted with a wind velocity of $U=$ $150 \mathrm{~km} / \mathrm{h}$, which was lower than the actual train speed because low turbulence and uniform flow were high priorities in this experiment. Velocity measurements were made using a Dantec 55P11 constant-temperature hotwire anemometer and linearizer. The hot wire was platinum with a diameter of $2.5 \mu \mathrm{m}$ and a length of $1.25 \mathrm{~mm}$, and the system frequency response was $5 \mathrm{kHz}$.

Figure 3 (a) shows the downstream development of velocity statistics in the boundary layer above the model surface, where $x$ is the distance from the nose to the observation point, $\delta$ is the boundary layer thickness defined as the distance required for the flow to reach $0.99 \mathrm{U}$, $\delta$ is the displacement thickness, $\sqrt{\overline{u_{0.4}^{\prime 2}}} / U_{\infty}$ represents the turbulence intensity at $0.4 \delta$, and $H_{12}\left(=\delta^{*} / \theta\right)$ is the shape factor defined by $\delta^{*}$ and the momentum thickness $\theta$. Figure 3 (b) shows the downstream development of intermittency factor $\Gamma$ in the boundary layer, representing the ratio

Table 1 Train model specifications

\begin{tabular}{c|l}
\hline Uniform diameter & $d=34.64 \mathrm{~mm}$ (about $1 / 110$ of full scale train) \\
\hline Nose/tail length & $l_{n}=51.96 \mathrm{~mm}$ \\
\hline Nose/tail shapes & Axisymmetric ellipsoid $2 l_{n} / d=3$ \\
\hline Overall length & $\begin{array}{l}\text { (i) } l_{r}=800 \mathrm{~mm} \text { (setup for wind tunnel) } \\
\text { (ii) } l_{T}=1300,2500 \mathrm{~mm} \text { (setup for model launch) }\end{array}$ \\
\hline Machined surface & Arithmetic average roughness Ra $=6.3 \mathrm{a}$ (JIS B 0601) \\
\hline Material & MC nylon polyamides 6 (MC901) \\
\hline
\end{tabular}

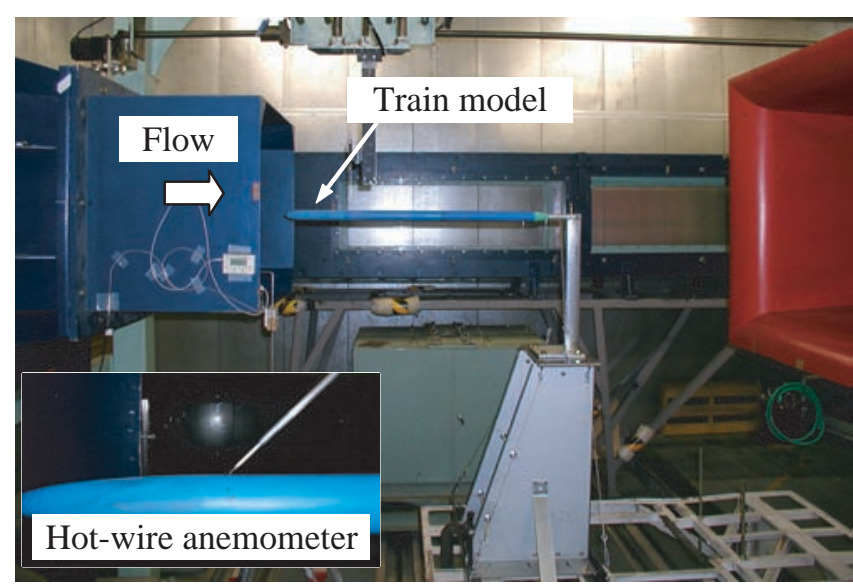

Fig. 2 Experimental setup of the axisymmetric model train in the wind tunnel facility

(a)

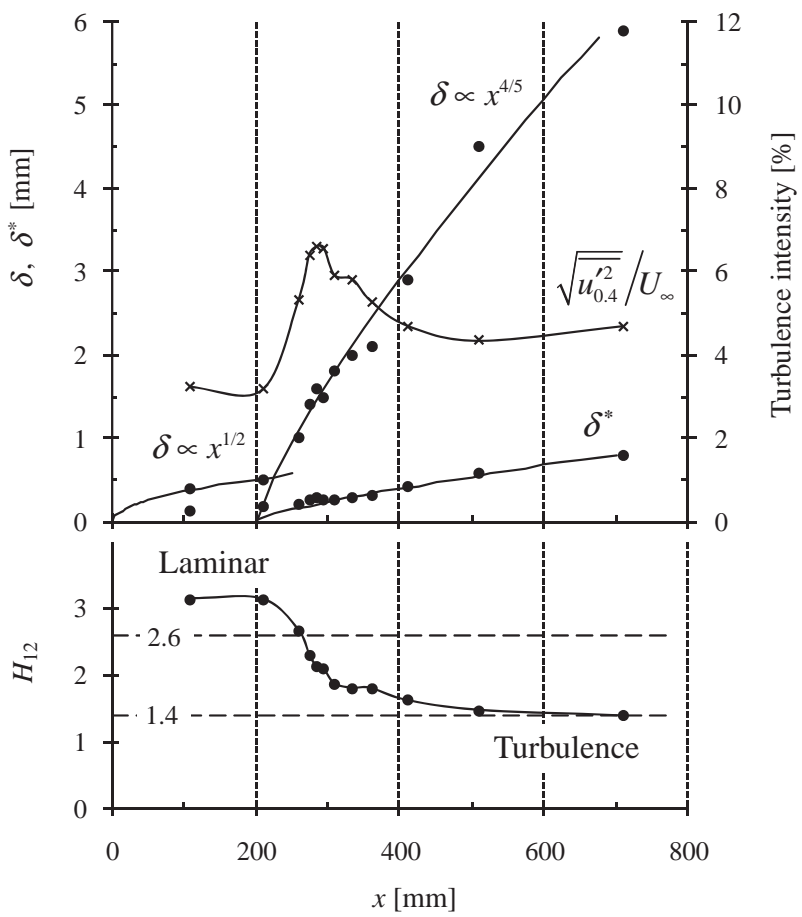

(b)

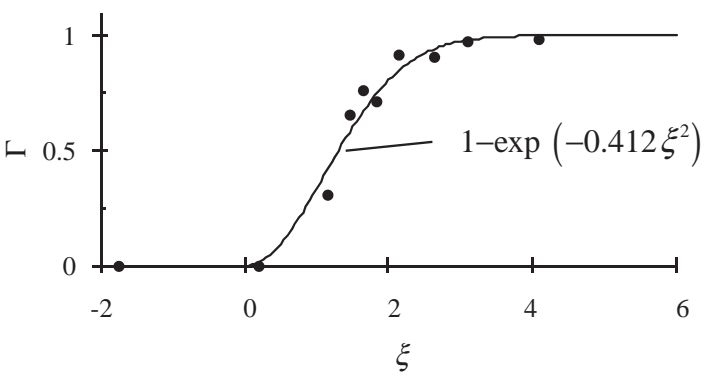

Fig. 3 Results of downstream development for (a) velocity statistics, and (b) intermittency factor

of laminar flow to turbulence flow; $\Gamma=1-\exp \left(-0.412 \xi^{2}\right)$ is the theoretical formula, where $\xi=\left(x-x_{0}\right) / x_{l}$ is the nondimensional length, $x_{0}$ is the transition point, and $x_{l}$ is 
the transition length [6].

The results here suggest that boundary layer transition to turbulence occurs at the point of $x \approx 0.2 \mathrm{~m}$ from the front of the model, where the Reynolds number based on the displacement thickness is about $\operatorname{Re}^{*}=500$. Then, the shape factor tends to fall off to an almost constant value of $H_{12}=14$, and the intermittency factor tends to rise to almost $\Gamma=1$, representing fully developed turbulence. The boundary layers above the model surface in a free-stream velocity of $U=150 \mathrm{~km} / \mathrm{h}$ or more therefore yield transition from laminar to turbulence at the point of $x \approx 0.2 \mathrm{~m}$ or less. These results were confirmed by spatial direct numerical simulation [7].

\subsection{Model launch experiment}

As the boundary layer of the model in a high-speed region is assumed to be almost turbulent (a significant candidate as a noise source), we investigated aerodynamic sound radiated from the same model using the model launch apparatus at the RTRI as shown in Fig. 4. This apparatus is usually used to investigate compression wave formation in tunnel conditions [8]. The train model is projected by means of a four-stage friction drive involving four pairs of vertically aligned wheels, and is guided along a 5 -mm-diameter taut steel wire. Small permanent magnets inserted into the model and two wire loops placed along the guide wire are used to measure the model's speed through magnetic field detection as it passes. The maximum possible launch speed is approximately $500 \mathrm{~km} / \mathrm{h}$. Pressure variations are measured by four precision sound-level meters (Rion NL-32, frequency response $20 \mathrm{~Hz}-20 \mathrm{kHz}$ ), at distances of $0.03 \mathrm{~m}, 0.1 \mathrm{~m}$, $0.2 \mathrm{~m}$ and 0.4 meters from the central axis of the model.

Figure 5 (a) shows the results of pressure variations obtained in the experiment. The experimental waveform is similar to the actual waveform of field measurement [4]. However, the magnitude of the experimental waveform of $P_{w, a}$ is much smaller than that of the actual waveform because of the difference between experimental and field conditions in scale.

Figure 5 (b) shows the power spectral density obtained in the experiment; the thin line indicates background noise from the launch apparatus, and the hatched area indicates sound above the background noise. There is thought to be a law of similarity for the relationship between the scale and the frequency. The typical frequencies of $P_{f}$ and $P_{w, a}$ are given by

$$
\begin{aligned}
& F_{f} \propto \frac{U}{r}, \\
& F_{w, a} \propto \frac{U}{\delta}
\end{aligned}
$$

where $F_{f}$ is a typical frequency for $P_{f}$ given approximately by potential theory [9], and $F_{w, a}$ is a typical frequency for $P_{w, a}$ estimated from the thickness of the turbulent boundary layer around the model as shown in Fig. 3.

Using (3) and (4), the typical sound frequencies of the model experiment can be converted to corresponding respective frequencies for real scale low-frequency noise. Comparing the calculated values of $F_{f}$ and $F_{w, a}$ listed in Table 2, the experimental frequencies are found to be close to the actual low-frequency noise obtained in past field measurements. Accordingly, the experiments using a simple-shape model can be thought to simulate the aerodynamically generated low-frequency noise of an actual train, and the turbulent boundary layers around the vehicle can be considered a key factor in determining the value of $P_{w, a}$.

Figure 6 (a) shows the distance attenuation of the magnitude of $P_{f}$ and $P_{w, a}$, where the horizontal axis represents the beeline distance from the central axis of the model to each microphone, and the magnitude of $P_{f}$ and $P_{w, a}$ are distinguished by an appropriate signal filter from the pressure waveform. According to the dominant frequencies estimated from the frequency analysis shown in Fig. 5 (b), the cut-off frequencies of the low-pass filter

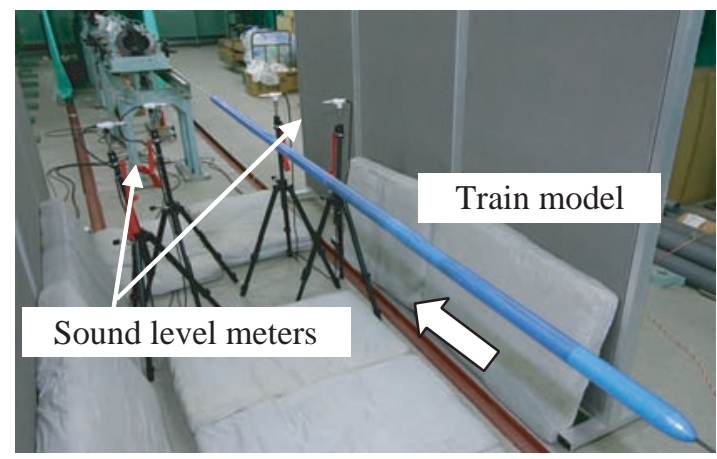

Fig. 4 Experimental setup of the axisymmetric model train at the model launch facility

(a)

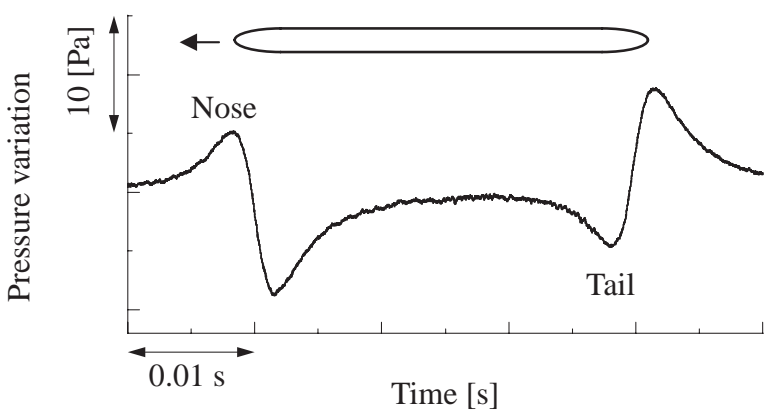

(b)

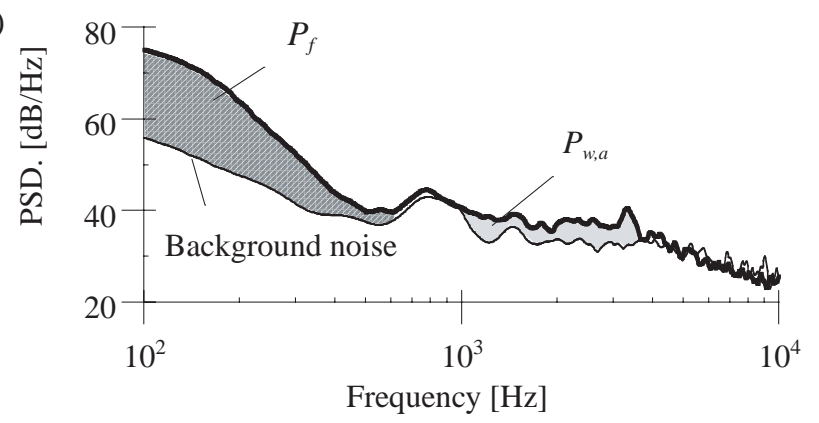

Fig. 5 Results of the model launch experiments for (a) the pressure waveform, and (b) power spectral density during the model's passage. The thin line indicates background noise from the launch apparatus, and the hatching indicates sound above the background noise. 
Table 2 Calculated frequencies from model experiment by Eqs. (3) and (4)

\begin{tabular}{c|c|c}
\hline & $F_{f}$ & $F_{w, a}$ \\
\hline \hline $\begin{array}{c}\text { Experimental results } \\
U=300 \mathrm{~km} / \mathrm{h}, r=0.2 \mathrm{~m}\end{array}$ & $<500 \mathrm{~Hz}$ & $1 \mathrm{k} \sim 4 \mathrm{kHz}$ \\
$\begin{array}{c}\text { Converted to actual frequencies } \\
U=270 \mathrm{~km} / \mathrm{h}, r=29 \mathrm{~m}\end{array}$ & $<3 \mathrm{~Hz}$ & $9 \sim 36 \mathrm{~Hz}$ \\
\hline
\end{tabular}

(a)

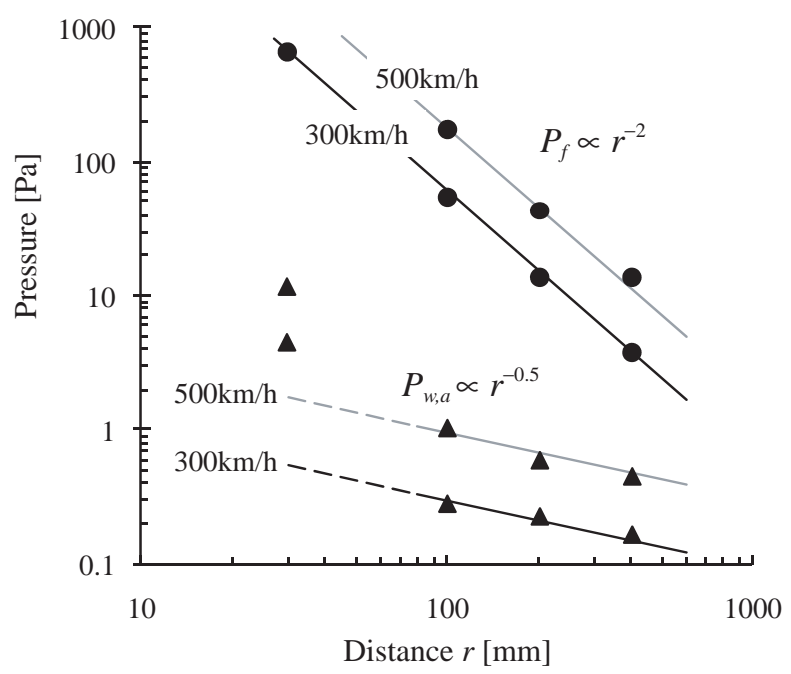

(b)

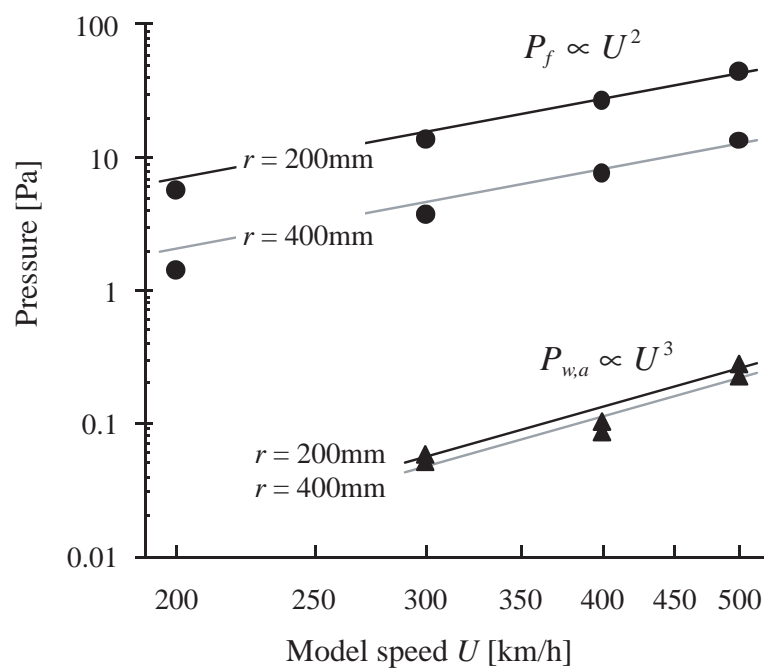

Fig. 6 Characteristics of the magnitude of $\boldsymbol{P}_{f}$ and $\boldsymbol{P}_{w, a}$ as obtained in the model launch experiment; (a) distance attenuation, and (b) velocity dependency

for $P_{f}$ were set to $500 \mathrm{~Hz}$, and those of the band-pass filter for $P_{w, a}$ were set to $800-5,000 \mathrm{~Hz}$. Since the waveform through the signal filter for $P_{f}$ exhibits a wave pattern showing a positive pulse followed by a negative pulse at the train nose part (their signs are reversed at the tail part), a magnitude of $P_{f}$ is defined as the difference between a positive pulse peak and a negative pulse peak at the nose part. However, the waveform through the signal filter for $P_{w, a}$ is observed as a continuous pressure wave while the train passes, so a magnitude of $P_{w, a}$ is defined as the RMS value during train passage.

As shown in Fig. 6 (a), the magnitude of $P_{f}$ is propor- tional to $r^{-2}$, and the magnitude of $P_{w, a}$ is proportional to $r^{-0.5}$. The distance attenuation of $P_{f}$ is equal to the coefficient of (1) obtained by potential theory, and the distance attenuation of $P_{w, a}$ is similar to the theoretically derived coefficient of the acoustic line source.

In addition, Fig. 6 (b) shows the velocity dependence of the magnitude of $P_{f}$ and $P_{w, a}$, where the horizontal axis represents the model's launch velocity. The results show that the magnitude of $P_{f}$ is proportional to $U^{2}$; this velocity dependence corresponds to the theoretical coefficient of (1). Meanwhile, the magnitude of $P_{w, a}$ is found to increase considerably in proportion to $U^{3}$, which suggests that $P_{w, a}$ is attributable to an aerodynamically generated dipole sound source [10].

\section{Field measurement}

Field measurement was performed at two Shinkansen sites close to a viaduct and an embankment in an openair section as shown in Fig. 7. Both tracks were straight, both roadbeds were of the non-ballasted slab type, and simple vertical sound barriers measuring about one meter in height or more were attached to both sides of the tracks. The measured train consisted of one set of six cars coupled with the other set of ten cars. Shapes and surface roughness of the two sets were basically the same, however the length of the cars and the wheelbase length of the bogies were different.

Low-frequency noise was measured with a specially manufactured sound-level meter for low-frequency (Rion $\mathrm{XN}-12 \mathrm{~A}$, frequency response: $0.2-1,000 \mathrm{~Hz}$ ), and the measurement points were located on the ground several
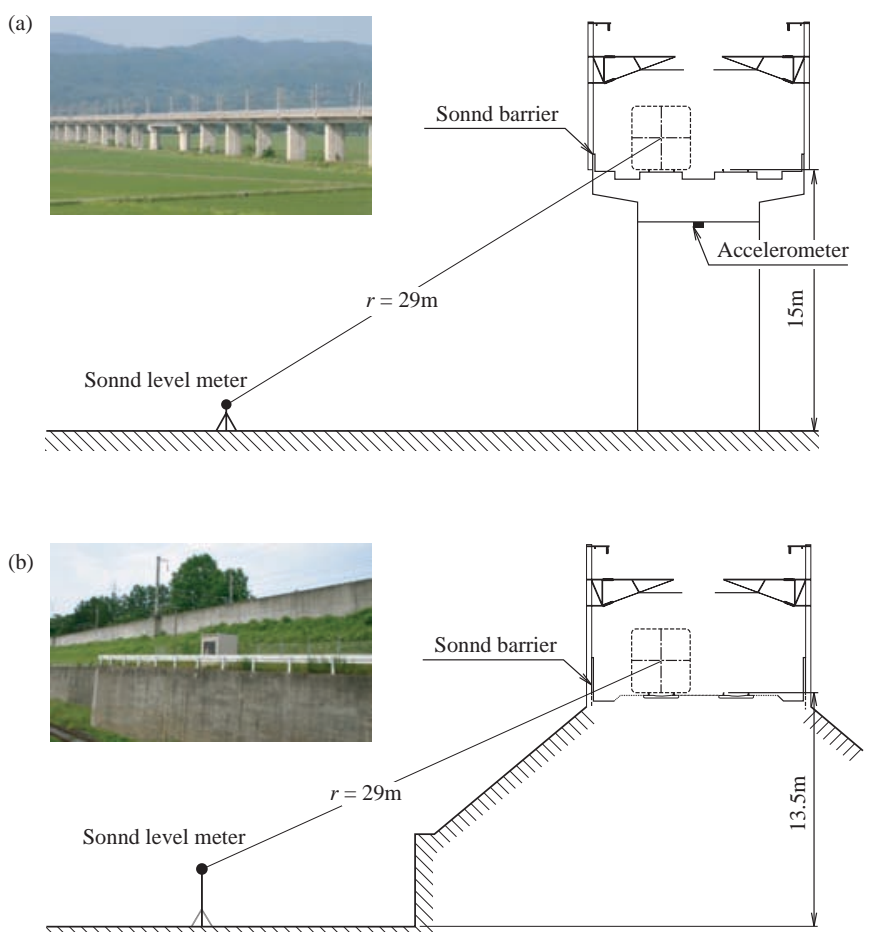

Fig. 7 Photograph and schematic images of the measurement sites in (a) the viaduct section, and (b) the embankment section 
dozen meters from the centerline of the down-track. In order to estimate the influence of noise radiation from the railway viaduct, vibration was also measured with an acceleration meter (Rion UV-05, frequency response: $1-10,000 \mathrm{~Hz}$ ) on the lower surface of a concrete beam. All measured data were recorded in time-history format on a digital data recorder (Sony PC-216Ax, frequency response: $\mathrm{DC}-5 \mathrm{kHz}$ ), from which it was possible to obtain a sound spectrogram and identify a coherence function between sound and vibration.

\subsection{Results of field measurement}

Figure 8 shows an ensemble averaged sound spectrogram with the same conditions at train speed $U=270$ $\mathrm{km} / \mathrm{h}$ and a measurement distance of $r \approx 29 \mathrm{~m}$, where the horizontal and vertical axes indicate the measurement time and frequency, respectively, the color shaded regions with color bar represent the magnitude of sound pressure level, and the frequency ranges of $F_{f}$ and $F_{w, a}$ are calculated from (3) and (4) by the typical frequency of the model experiment.

In these figures, infrasound values with a frequency less than $3 \mathrm{~Hz}$ are attributed to the $P_{f}$ phenomenon, or pressure variations caused by moving quasi-steady pressure fields around the nose and tail parts of the train. Broadband noise in the range of 10 to $40 \mathrm{~Hz}$ is attributed to the $P_{w, a}$ phenomenon, or noise aerodynamically

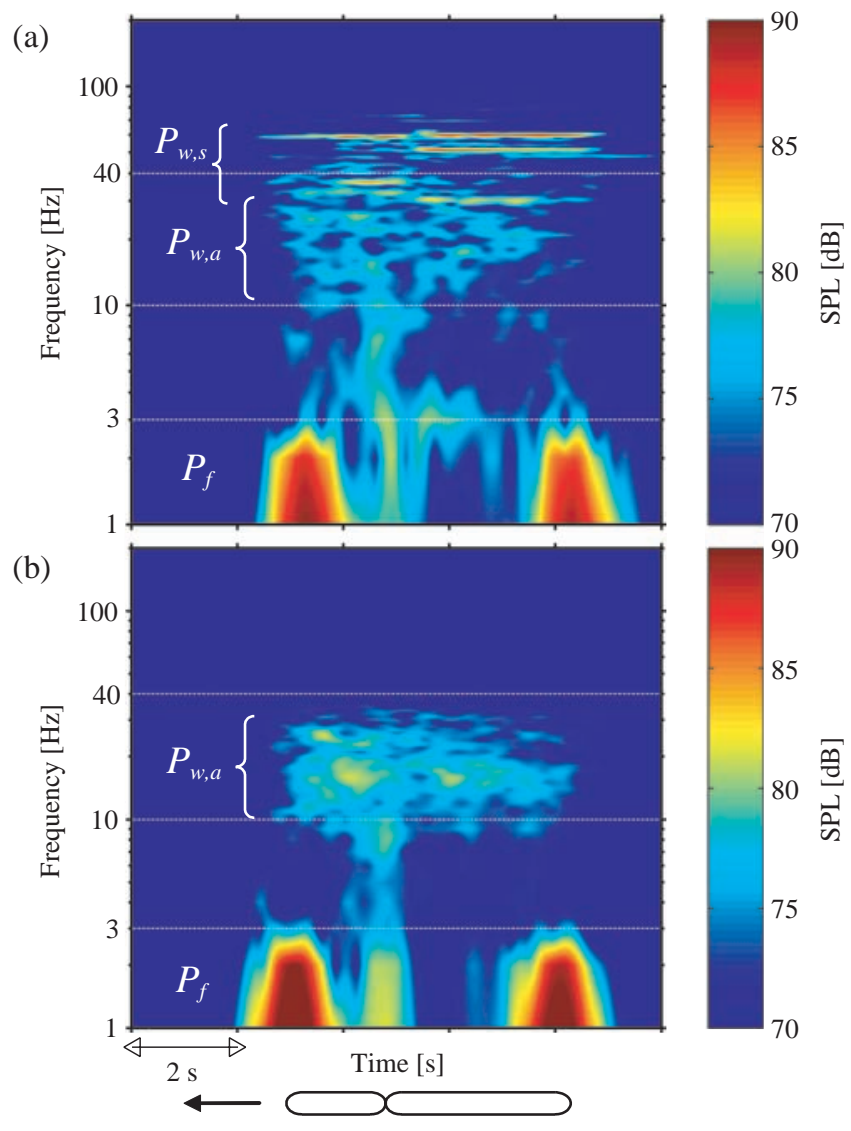

Fig. 8 Spectrograms of sound pressure in (a) the viaduct section, and (b) the embankment section; $U=270$ $\mathrm{km} / \mathrm{h}, r \approx 29 \mathrm{~m}$ generated from the train. In addition, for the viaduct section, several periods of continuous noise with a frequency less than $80 \mathrm{~Hz}$ added to $P_{w, a}$ are attributed to the $P_{w, s}$ phenomenon, or noise radiation from concrete railway viaducts. This is confirmed by the fact that the coherence function between sound and viaduct vibration is relatively high in accordance with the frequencies of $P_{w, s}$. Note that the frequencies of $P_{w, s}$ at the front of the train are different from those at the rear because different vibration modes of the viaduct structure are caused by differences in the car length and the wheelbase length of the two sets.

\subsection{Characteristics of low-frequency wayside noise}

Figure 9 shows the actual measurements and the characteristics of low-frequency wayside noise estimated from both the field measurements and the model experiment, where $L$ is the maximum sound pressure level (SPL) of low-frequency noise (F-weighted: flat in $1-100 \mathrm{~Hz}$ [11]) with a constant of $0.125 \mathrm{~s}$ for time weighting. Additionally, $L$ is divided into the three categories of $L_{f}$ (the SPL of $\left.P_{f}\right), L_{w, s}$ (the SPL of $P_{w, s}$ ) and $L_{w, a}$ (the SPL of $P_{w, a}$ ). A near field and a far field are distinguished by $r / d \lessgtr 12$ (where $d$ is the equivalent circle diameter of the train cross-section) because the magnitude of $L_{f}$ is related to $r$,

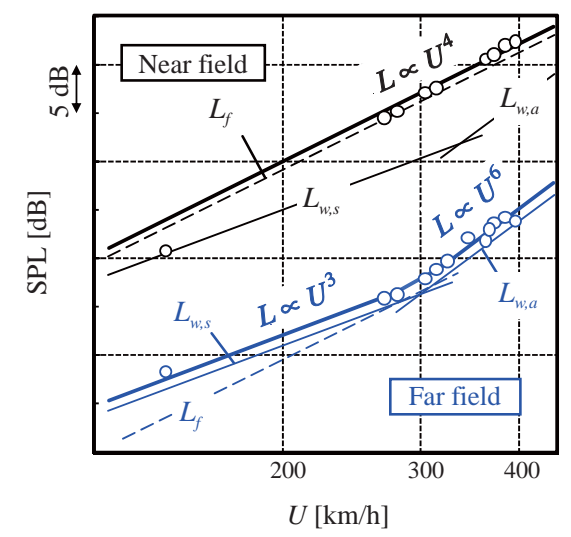

(a) Viaduct section

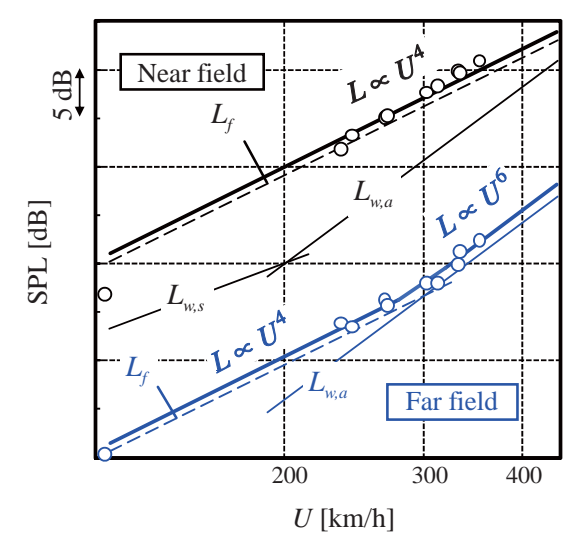

(b) Embankment section

Fig. 9 Characteristics of low-frequency wayside noise for near field $(r=15 \mathrm{~m})$ and far field $(r=45 \mathrm{~m})$. Symbols "O" represent measured maximum sound pressure levels; lines are estimated characteristics of low-frequency wayside noise 
the measurement distance, and $A_{0}=\pi(d / 2)^{2}$, the crosssectional area of the train, by (1).

By comparing the near field with the far field, it can be seen that $L_{f}$ significantly affects the magnitude of $L$ in the near-field range, and the influence of the type of site is negligible. Therefore, the low-frequency wayside noise of both the viaduct section and the embankment section tend to increase with $L \approx L_{f} \propto U^{4}$.

By contrast, the magnitude of $L$ in the far-field range is affected by the type of site and the train's speed range. For speeds of around $300 \mathrm{~km} / \mathrm{h}$ or less, low-frequency wayside noise in the viaduct section is larger than that in the embankment section in the far-field range because of the effect of $L_{w, s}$ of the concrete structure. For speeds of around $300 \mathrm{~km} / \mathrm{h}$ or higher, $L_{w, a}$ gradually affects the magnitude of $L$, and both low-frequency wayside noise in the viaduct section and that in the embankment section tend to increase with $L \approx L_{w, a} \propto U^{6}$.

\section{Conclusions}

In this paper, the three major noise source mechanisms of high-speed trains have been discussed; pressure variations around the nose and tail parts of the train generated by train passage, aerodynamically generated low-frequency pressure waves emanating from the train itself, and noise radiation from vibrating concrete railway viaducts.

The measurement of higher-speed region showed that the most significant source of low-frequency noise in the far field is aerodynamically generated unsteady flow, which is analogous to a line source. Laboratory experiments using an axisymmetric train model indicated that the turbulent boundary layer developing on the train surface is a key issue in the mechanism behind low-frequency wayside noise.

\section{Acknowledgment}

The authors would like to thank Prof. Hiroshi Maekawa of the University of Electro-Communications for his advice, and are also grateful to Mr. Takeshi Kurita and the staff of the JR East Group's Research and Development Center for their indispensable work in the vehicle running tests.

\section{References}

[1] Kikuchi, K., Iida, M., Takasaki, T., and Takami, H., "Field measurement of wayside low-frequency noise emitted from tunnel portals and trains of high-speed railway," Journal of Low Frequency Noise, Vibration and Active Control, Vol. 24, No. 4, pp.219-231, 2005.

[2] Ministry of the Environment of Japan, "Manual for measurement of low-frequency noise," 2000 (in Japanese).

[3] Kikuchi, K. and Iida, M., "Simple numerical method to calculate pressure fluctuation due to train passage," Transactions of the Japan Society of Mechanical Engineers, Series B, Vol. 71, No. 708, pp. 2022-2029, 2005 (in Japanese).

[4] Takami, H., Kikuchi, K., Maekawa, H., Kurita, T., and Wakabayashi, Y., "Low-frequency noise radiated from a high-speed train passing an open section," Transactions of the Japan Society of Mechanical Engineers, Series B, Vol. 73, No. 735, pp. 2275-2282, 2007 (in Japanese).

[5] Nagakura, K. and Zenda, Y., "Prediction model of wayside noise level of Shinkansen," proceeding of International Workshop Wave 2002, pp. 237-244, 2003.

[6] Dhawan, S. and Narashimha, R., "Some properties of boundary layer flow during the transition from laminar to turbulent motion," Journal of Fluid Mechanics, Vol. 3, pp. 418-436, 1958.

[7] Watanabe, D., Takami, H., Maekawa, H., Kikuchi, K., Iida, M., and Suzuki, H., "Acoustic waves emanating from transitional structures in a compressible boundary-layer for high-speed train," presented at the Thirteenth International Congress on Sound and Vibration, 2006.

[8] Ozawa, S. and Maeda, T., "Model experiment on reduction of micro-pressure wave radiated from tunnel exit," presented at International Symposium on Scale Modeling, Japan Society of Mechanical Engineers, Tokyo, July 18-22, 1988.

[9] Howe, M. S., "On the infrasound generated when a train enters a tunnel," Journal of Fluids and Structures, Vol. 17, pp. 629-642, 2003.

[10]Curle, N., "The influence of solid boundaries upon aerodynamic sound," Proceedings of the Royal Society of London, Series A, Vol. 231, No. 1187, pp. 505-514, 1955.

[11]Iwahashi, K. and Ochiai, H., "Infrasound pressure meter and examples of measuring data," Journal of Low Frequency Noise, Vibration and Active Control, Vol. 20, No. 1, pp. 15-19, 2001. 